\title{
Técnicas de Modelagem de Conformação de Chapas por Elementos Finitos
}

\author{
Professor Mestre Alexandre Alvarenga Palmeira (Engenharias - UERJ) - \\ palmeira@uerj.br \\ Professor Mestre Alexandre Fernandes Habibe (Engenharias - UniFOA) - \\ alexandre.habibe@foa.org.br \\ Professor Mestre Paulo André Dias Jacome (Engenharias - UniFOA) - \\ paulo.andre@foa.org.br
}

\section{Resumo}

A necessidade de redução do tempo e dos custos de geração de um projeto faz com que a indústria utilize métodos de simulação numérica para o desenvolvimento de produtos. $O$ objetivo deste trabalho é avaliar quais os modelos e métodos de solução são mais adequados e eficientes na simulação da conformação de chapas pelo MEF, através de modificações dos parâmetros de simulação, tais como: técnica de integração no tempo, velocidade de análise e refino de malha. Para tanto, é simulado o ensaio de estiramento de chapas finas proposto pela Universidade do Estado de Ohio e o embutimento de uma lata de atum. Nas simulações é utilizado o código ABAQUS. Observa-se, que o método implícito dinâmico possuir uma melhor precisão nos resultados simulados, quando comparados com os resultados experimentais e apresenta um elevado custo computacional. Em virtude de tal fato, o método explícito dinâmico apresenta uma melhor relação custo/benefício. Conclui-se que a velocidade imposta ao modelo, apresenta uma grande influência na acurácia dos resultados. Observa-se que mesmo para níveis de velocidade muito baixos a relação energia cinética/energia de deformação é superior a 5\%. Outro aspecto que apresenta elevada influência na precisão dos resultados é o refino da malha da chapa.

Palavras Chaves: Estampagem, Estiramento, ABAQUS.

\section{Introdução}

A conformação chapas consiste em transformar uma chapa plana em um produto com formato desejado sem que ocorra fratura ou redução excessiva da espessura. Os processos de conformação de chapas podem ser simples como um dobramento, ou muito complexos como em estampagens de rodas automobilísticas, mas sempre a conformação consistirá de uma seqüência de operações de dobramento, estiramento, estampagem profunda ou embutimento. Levando em consideração estes aspectos são simulados neste trabalhos dois processos: ensaio de estiramento de chapas finas, proposto pela Universidade do Estado de Ohio, e de um embutimento de uma lata de atum. 


\section{Ensaio de Estiramento}

O estiramento é o processo de conformação que consiste na aplicação de forças de tração de maneira a esticar o material sobre uma ferramenta ou bloco-modelo ${ }^{(1)}$. O estiramento de uma chapa ocorre quando aplicam-se tensões de tração ao plano da chapa. Vários ensaios foram desenvolvidos com o objetivo de simular a capacidade de estiramento dos materiais. Dentre eles destacam-se: Ensaio de Erichsen/Olsen e o Ensaio da Altura Limite do Domo (Hecker/Ghosh) ${ }^{(2)}$. Recentemente Miles et al ${ }^{(3)}$, propuseram um novo ensaio de estiramento que fornecesse um estado de deformação plana de maior reprodutibilidade e que apresentasse um menor tempo de execução em relação ao ensaio da altura limite do domo. Este ensaio é chamado de ensaio de conformabilidade em deformação plana ou "Ensaio de Conformabilidade da Universidade de Ohio" (OSU Formability Test). A geometria final deste ensaio obtida após comparações com outras geometrias alternativas e otimizada pela simulação através do MEF é apresentada na Figura $1^{(4)}$.

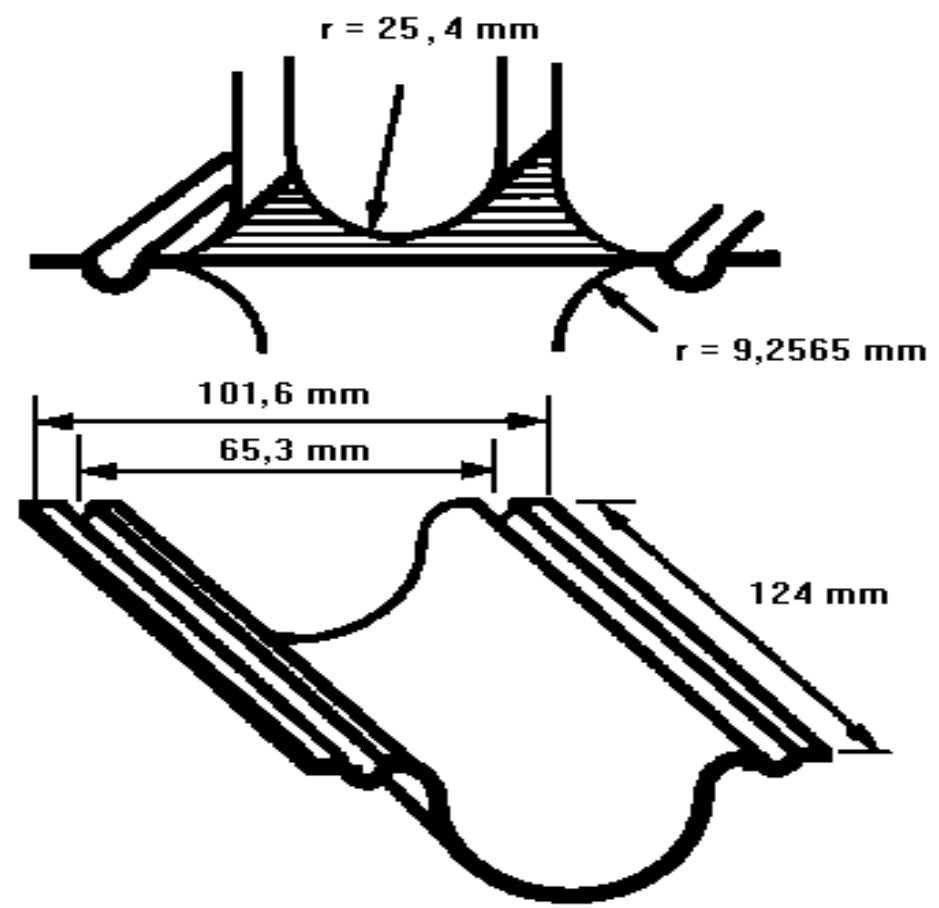

Figura 1: Geometria do Ensaio de Conformabilidade da Universidade de Ohio.

\section{Estampagem Profunda}

Esta operação diferencia-se da operação de estiramento, por permitir que as laterais da chapa escoem para o interior da matriz, fazendo com que praticamente não ocorra variação da espessura. Na operação de embutimento cilíndrico, este processo caracteriza-se por utilizar um punção cilíndrico que irá formar um copo como produto final. Durante a formação do copo, o material do esboço ou material de partida é conformado para o interior da matriz, passando por vários níveis e estados de tensões diferentes até que todo o material esteja colocado no interior da matriz e o copo formado. O prende-chapas é utilizado para evitar o enrugamento no flange, provocado pelas tensões de compressão que surgem pela redução do diâmetro. Porém, a força que o prende-chapas exerce não impede o deslocamento das bordas da chapa e apenas deve manter seu flange plano ${ }^{(5 ; 6 ; 7)}$. 
Existem duas regiões críticas na estampagem de copos: o flange, onde a maior parte da deformação ocorre, e a região de concordância entre a parede e o fundo do copo que deve suportar uma força suficiente para causar a deformação no flange. Se o diâmetro do "blank" (esboço) é muito grande, a força que deve ser transmitida pela parede será excessiva, causando portanto, escoamento e ruptura na mesma ${ }^{(5)}$.

\section{Simulação Numérica}

A necessidade de redução do tempo e dos custos de geração de um projeto leva a indústria a utilizar métodos de simulação numérica para o desenvolvimento de seus produtos. A equação de governo que rege o equilíbrio em uma análise linear dinâmica no sistema de elementos finitos, é dada por:

$$
M \ddot{U}+C \dot{U}+K U=R
$$

onde $M, C$ e $K$ são as matrizes de massa, amortecimento e rigidez respectivamente, e $\ddot{U}$, $\dot{U}$ e $U$ correspondem aos vetores de aceleração, velocidade e deslocamentos respectivamente e $R$ o vetor de carregamento externo. De acordo com Westheiner et al, citado por Menezes ${ }^{(8)}$, as técnicas numéricas de integração são classificadas em método de integração implícito e explícito no tempo, devido às equações de governo serem integradas, ambas, no espaço e no tempo.

\subsection{Método Explícito de Integração no Tempo}

De acordo com Schweizerhof et al $^{(9)}$ a integração explícita no tempo é para problemas específicos, pois é mais eficiente uma vez que integra a variável tempo em conjunto com o espaço discretizado por elementos finitos em problemas de mecanismos estruturais que dependem do tempo. É interessante notar que este método de integração não necessita de uma fatorização efetiva da matriz de rigidez a cada passo, fato este que é a chave de sua eficiência computacional, a matriz de massa $M$ é dita concentrada ("lumped mass") ${ }^{(10)}$.

O uso do procedimento explícito requer um tempo de incremento muito pequeno, pois o método de integração faz necessário o uso de um "time step"- $\Delta t$ que deve ser menor que um valor crítico - $\Delta t_{c r}$ que pode ser calculado em função das propriedades características do sistema (rigidez, amortecimento, massa específica, etc.). Este parâmetro limite de estabilidade, $\Delta t_{c r}$, é dado em termos dos autovalores do sistema, isto é:

$$
\Delta t_{c r} \leq \frac{2}{w_{\max }}
$$

Segundo Rebelo et $a l^{(11)}$, o limite de estabilidade pode ser aproximado pelo menor tempo necessário para que uma onda elástica atravesse qualquer elemento da malha. Em uma análise que dure $T$ segundos, o programa deve executar $N$ incrementos com duração de $\Delta t$ segundos cada um, ou seja, o número total de incrementos, $N$, requeridos para completar a análise é dado por $T / \Delta t$. De acordo com Rebelo et al ${ }^{(11)}$, no procedimento explícito o incremento de tempo estável $\left(\Delta t_{c r}\right)$ é tipicamente muito pequeno se comparado com o tempo natural de duração do processo de conformação. Qualquer abordagem para redução do tempo de processamento requerido para analisar uma dada malha precisará aumentar o incremento de tempo $\Delta t$ ou reduzir o tempo total $T$, ou seja, a velocidade do ferramental pode ser aumentada, através do aumento da velocidade do punção (load factoring). 


\subsubsection{Load Factoring}

Consiste em se reduzir o tempo total de análise $T$, simplesmente diminuindo o tempo da aplicação de cargas e das condições de contorno. A vantagem deste método, é que as propriedades do material permanecem inalteradas, contudo esta abordagem, pode também fornece resultados errados se a velocidade do processo for aumentada além de certos limites ${ }^{(12)}$. Com o aumento da velocidade dos componentes a energia cinética também aumenta e pode eventualmente atingir a mesma ordem de grandeza da energia de deformação ou do trabalho feito no material. Não é fácil prever em que nível este efeito é significativo, mas uma regra baseada na experiência é limitar a energia cinética em no máximo 5\% da energia de deformação ${ }^{(12)}$. Segundo Di Pasquale et al ${ }^{(13)}$, experimentos mostram que a velocidade do punção, em alguns casos, pode alcançar cerca de 15 a $20 \mathrm{~m} / \mathrm{s}$ antes que os efeitos de inércia sejam sentidos nos resultados, principalmente na estampagem.

\subsection{Método Implícito de Integração no Tempo}

Este método é assim classificado por que a solução da equação de governo que rege o equilíbrio é obtida pela consideração no tempo $t+\Delta t$. Sendo conhecidos o deslocamento e a velocidade no tempo $t$, para obter estes valores no tempo $t+\Delta t$, o equilíbrio de forças no tempo é dado pela equação (3):

$$
M \ddot{U}_{t+\Delta t}+C \dot{U}_{t+\Delta t}+K U_{t+\Delta t}=R_{t+\Delta t}
$$

O procedimento empregado para solucionar a equação (3) é o método de Newmark de integração no tempo ${ }^{(14)}$, que utiliza uma expansão em diferenças finitas no intervalo de tempo $\Delta t$ para aproximar os valores de $U_{t+\Delta t}$ e $\dot{U}_{t+\Delta t}^{(15)}$. Após este procedimento, em problemas que envolvem não-linearidades, geralmente é utilizado o método incremental de Newton-

Raphson $^{(14)}$ para se obter o valor de $\ddot{U}_{t+\Delta t}$. Nele o carregamento é prescrito, por exemplo o deslocamento do punção é dividido em pequenos incrementos e em cada um destes é calculado o vetor deslocamento. Este método requer a montagem e a inversão da matriz de rigidez a cada novo incremento, o que representa um aumento no tempo de processamento e uma maior requisição de memória na análise de modelos tridimensionais.

\section{Elementos e sua Disposição no Modelo}

Os elementos utilizados na simulação dos processos de conformação de chapas devem apresentar características que os tornem capazes de resistir aos diversos tipos de solicitações a que são submetidos durante o processo de conformação, dobramento, estiramento e embutimento. No processo de conformação de chapas um aspecto de vital importância a ser considerado é a deformação elasto-plástica a qual a chapa é submetida durante o dobramento (flexão) e o estiramento. Devido à tensão transversal normal ser secundária, a cinemática considerada na teoria clássica de cascas pode ser utilizada ${ }^{(9)}$.

Outro fator que pode tornar onerosa a análise é o número de elementos da malha, pois o tempo de processamento é proporcional ao número de elementos presentes no modelo. Burford et al $^{(16)}$ observou que os resultados simulados, tanto de medidas de deformação quanto de força, são bastante sensíveis não só ao refinamento da malha mas a 
disposição dos elementos na mesma. Mattiasom et $a l^{(17)}$ relacionou o tamanho do elemento ao tamanho do menor raio (raio de adoçamento do punção) presente num processo de estampagem. Ele concluiu que o tamanho de elemento ideal deve ser igual a metade do menor raio presente na geometria do modelo e que o nível de oscilação de forças presentes nos resultados simulados aumenta com o tamanho do elemento.

\subsection{Formulação do Contato}

Nas análises de conformação mecânica é muito importante e complexa a modelagem do contato entre a ferramenta e a peça que está sendo trabalhada, pois considera-se possível a interferência, o deslizamento e a perda do contato das mesmas durante a simulação. Na modelagem matemática, adotada no ABAQUS, dependendo da natureza do problema de contato, dois procedimentos podem ser aplicados:

\subsubsection{Superfícies de Contato}

Para a estratégia de contato, a superfície cujos nós são testados contra a penetração é chamada de superfície "master" e a do nó penetrante é chamada de "slave". Neste modelo, cada potencial de contato tem suas condições definidas em termos de duas superfícies em contato. Os nós da superfície slave são restringidos para não penetrar na superfície master. Consequentemente a direção de contato é sempre normal à superfície master. Os nós da superfície master podem, em princípio, penetrar na superfície slave ${ }^{(18)}$.

Neste tipo de formulação, todas as partes que compõem o ferramental fazem uso de uma formulação de corpo rígido (master) que possui na sua constituição elementos que não se deformam quando entram em contato com outras superfícies (slave). O código de elementos finitos ABAQUS faz uso de dois tipos de formulação de superfície de contato, e são elas: RIGID BODY (Corpo Rígido) e RIGID SURFACE (Superfície Rígida).

\section{a) RIGID BODY - Corpo Rígido ${ }^{(19)}$}

Este tipo de formulação é aplicada, em geral, em geometrias tridimensionais. Esta técnica consiste em se criar a geometria do ferramental (master) e utilizar elementos de formulação rígida para compor a mesma. Cada superfície gerada possui um nó de referência onde são aplicas as restrições cinemáticas de movimento do modelo. No nó de referência também são aplicados carregamentos concentrados. Nos demais nós do modelo podem ser aplicados carregamentos distribuídos, como pressão e forças de corpo. Apesar desta formulação ser utilizada no método implícito e explícito de integração no tempo, ela apresenta diferenças. No método implícito de integração no tempo, o código ABAQUS aplica as restrições de modo que os nós da superfície slave não penetrem na superfície master, mas em princípio, os nós da superfície master podem penetrar na superfície slave. Já quando o código utiliza o método explícito de integração no tempo, a formulação master-slave é "estimada". Neste caso, as restrições de contato são aplicadas de modo que não ocorra penetração em ambas as superfícies, ou seja, o contato é forçado de modo que as duas superfícies façam o papel de superfície master e slave ao mesmo tempo. Impedindo assim que os nós da superfície slave penetrem nos segmentos da superfície master e vice-versa. As restrições de contato são, desta forma, uma média das duas restrições master-slave. 


\section{b) RIGID SURFACE - Superfície Rígida ${ }^{(20)}$}

Esta formulação é utilizada para definir superfícies geométricas simples, pela descrição de sua seção transversal com segmentos de linhas e retas. Neste caso é definido o contorno da superfície a ser gerada e o código gera a superfície com seus respectivos elementos através de critérios internos de escolha, sem que se possa alterar o tipo ou o número de elementos que compõem a superfície. Esta formulação é computacionalmente menos custosa que a formulação anterior. Assim a superfície master gerada possui um nó de referência no qual são aplicados os carregamentos e as restrições cinemáticas do modelo. Esta formulação, no código ABAQUS, só pode ser utilizada em conjunto com o método implícito de integração no tempo.

\section{Resultados e Discussões}

\subsection{Dados Experimental}

Os resultados experimentais utilizados, tanto na simulação numérica (propriedades intrínsecas dos materiais - dados de entrada) quanto os resultados comparados com os dados simulados (espessura, deformações, etc.) foram obtidos dos trabalhos desenvolvidos por Moreira $^{(2)}$ e Villas Boas ${ }^{(21)}$.

Moreira $^{(2)}$ realizou ensaios experimentais de estiramento para o material folha de flandres, espessura inicial de $0,22 \mathrm{~mm}$, os resultados foram avaliados em ensaio sem lubrificação (seco). Moreira ${ }^{(2)}$ fez uso de esboços metálicos com formato retangular cujas dimensões são: $200 \mathrm{~mm}$ por $120 \mathrm{~mm}$, com a maior dimensão paralela à direção de laminação. As distribuições das deformações foram obtidas através da análise das circunferências (grafadas inicialmente na chapa), após a realização do ensaio, onde o ponto 0 representa o polo ou o ponto que passa pela linha de centro e simetria na direção da largura do esboço. A distribuição das deformações e a variação de espessuras são tomadas ao longo da linha de centro na direção do comprimento do esboço, a direita do ponto 0 .

Já Villas Boas ${ }^{(21)}$ realizou experimentalmente a estampagem de um copo cilíndrico para o material folha de flandres, espessura inicial de $0,24 \mathrm{~mm}$. O embutimento foi realizado em um esboço com formato circular e com $148 \mathrm{~mm}$ de diâmetro. As distribuições das deformações são obtidas através da análise de circunferências, previamente serigrafadas no modelo antes da estampagem, após a realização da estampagem. As medidas são tiradas no eixos principal da circunferência deformada em função da distância (perímetro) ao longo de meia seção axial da lata, tomando-se como referência a direção de laminação (direção de $0^{\circ}$ ).

\subsection{Métodos de Integração no Tempo}

O presente trabalho tem como um dos objetivos avaliar e comparar os resultados experimentais do ensaio de estiramento e da estampagem de uma lata de atum com os resultados obtidos através de simulações utilizando o código de elementos finitos ABAQUS, na versão ABAQUS/STANDART (implícita) e na versão ABAQUS/EXPLICT (explícita). Todas as simulações foram realizadas em três dimensões (ensaio de estiramento) e em duas dimensões com um modelo axisimétrico (processo de estampagem) em um supercomputador CRAY J90. 


\subsubsection{Modelo de Estiramento}

São realizadas simulações em três dimensões, utilizando o método implícito dinâmico e explícito dinâmico de integração no tempo os resultados são resumidos na Tabela 1.

\begin{tabular}{l|c|c|c|c}
\hline \hline $\begin{array}{c}\text { Método de } \\
\text { Integração }\end{array}$ & $\begin{array}{c}\text { Tempo de } \\
\text { Processamento } \\
\text { (s) }\end{array}$ & $\begin{array}{c}\text { Número de } \\
\text { Incrementos }\end{array}$ & $\begin{array}{c}\text { Deformação } \\
\text { Crítica } \\
(\mathbf{m m})\end{array}$ & $\begin{array}{c}\text { Coordenada da } \\
\text { Def. Crítitca } \\
\text { (mm) }\end{array}$ \\
\hline \hline Explícito & $2.180,01$ & 53.100 & 0,16197 & 18,264 \\
\hline Implícito & $7.916,62$ & 512 & 0,17284 & 18,239 \\
\hline \hline
\end{tabular}

Tabela 1: Resultados obtidos na simulação do ensaio de estiramento.

Observa-se que apesar de ter sido imposta a mesma velocidade ao ferramental $(1 \mathrm{~m} / \mathrm{s})$ para ambas as análises o tempo de processamento consumido pelo método implícito dinâmico é 3,7 vezes superior ao tempo de processamento do método explícito dinâmico. Observa-se também que o número de incrementos utilizados pelo método explícito dinâmico é 100 vezes superior ao utilizado pelo método implícito dinâmico.

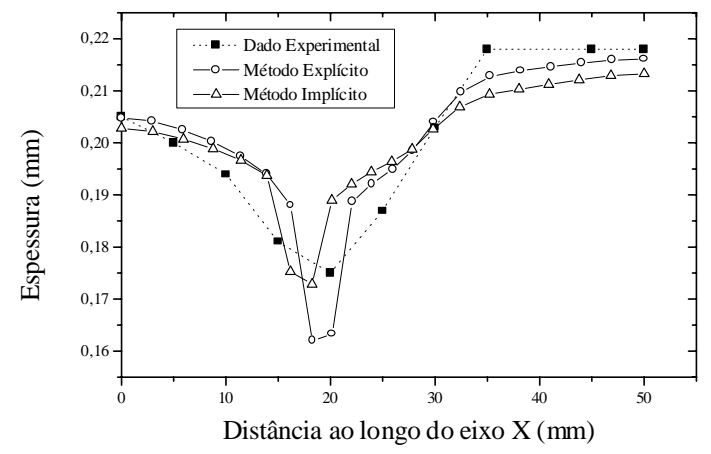

(a)

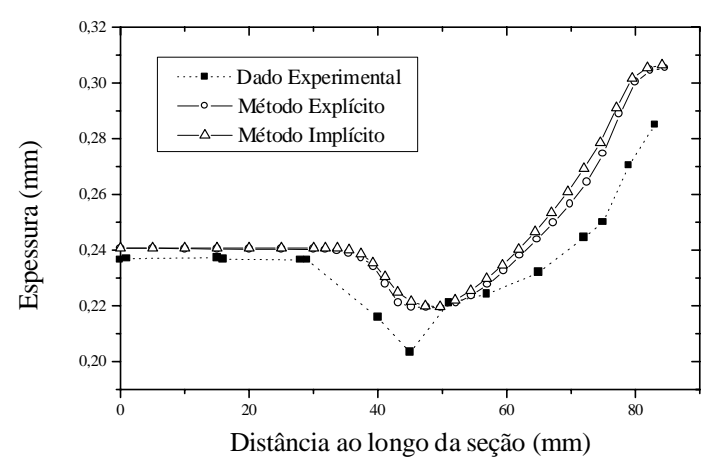

(b)

Figura 2: (a) Distribuição de espessuras no ensaio de estiramento;

(b) Distribuição de espessuras no processo de estampagem.

Com relação a acurácia dos resultados de simulação (Figura 2a), pode-se observar que o maior afinamento previsto pelo método implícito dinâmico é 6,7\% maior do que o previsto pelo método explícito dinâmico de integração no tempo. Apesar desta diferença, os dois métodos de integração no tempo apresentam uma boa concordância qualitativa com a curva experimental, onde o método implícito dinâmico obtêm resultados mais próximos ao resultado experimental, fato este observado na Figura 2a.

\subsubsection{Modelo de Estampagem da Lata da Atum}

Assim como no modelo anterior, são realizadas simulações do processo de estampagem da lata de atum através do método explícito dinâmico e implícito dinâmico de 
integração no tempo, mas em duas dimensões com um modelo axisimétrico, cujos resultados são resumidos na Tabela 2 e analisados, a seguir.

\begin{tabular}{l|c|c|c|c}
\hline \hline $\begin{array}{c}\text { Técnica de } \\
\text { Integração }\end{array}$ & $\begin{array}{c}\text { Tempo de } \\
\text { Processamento } \\
\text { (s) }\end{array}$ & $\begin{array}{c}\text { Número de } \\
\text { Incrementos }\end{array}$ & $\begin{array}{c}\text { Deformação } \\
\text { Crítica } \\
(\mathbf{m m})\end{array}$ & $\begin{array}{c}\text { Coordenada da } \\
\text { Def. Crítitca } \\
\text { (mm) }\end{array}$ \\
\hline \hline Explícita & $2.294,81$ & 124.801 & 0,21922 & 79,773 \\
\hline Implícita & $24.579,21$ & 5.556 & 0,21968 & 79,638 \\
\hline \hline
\end{tabular}

Tabela 2: Resultados obtidos na simulação do processo de estampagem.

Pode-se observar que, assim como no caso anterior, o método implícito dinâmico consome um maior tempo de processamento (cerca de 10 vezes superior ao tempo de processamento do método explícito) mas necessita de cerca de um vigésimo no número de iterações gastas pelo método explícito para chegar ao final da análise fazendo uso da mesma velocidade imposta ao ferramental.

Na Figura 2b, observa-se em ambos os métodos utilizados uma boa concordância qualitativa, onde a maior diferença para ambas as simulações foi de $10 \%$ na espessura, na região da parede da lata. Para ambos os métodos a maior diferença foi de $8 \%$ no ponto de maior afinamento. É importante ressaltar que os resultados simulados pelos métodos implícito dinâmico e explícito dinâmico praticamente apresentaram coincidência total dos valores simulados de distribuição de espessura.

\subsubsection{Disposições Finais}

Ambos os modelos, tanto para ensaio de estiramento quanto para o processo de estampagem, o método implícito dinâmico mostrou-se ser mais estável, uma vez que faz uso de tempos de incremento ( $\Delta t)$ maiores nos passo de integração (menor número de incrementos). Por outro lado mostrou-se ser mais custoso (oneroso), pois consumiu maiores tempos de processamento, chegando a ser até 10 vezes superior ao tempo gasto pelo método explícito dinâmico (modelo de estampagem). Esta grande diferença no tempo de processamento gasto pelo método implícito é causado pelo fato de que a cada novo incremento este método requer a montagem e a inversão da matriz de rigidez ${ }^{(14)}$. O método explícito de integração no tempo faz uso de uma matriz de massa diagonal, dispensando assim o uso de qualquer método de decomposição ou inversão de matrizes ${ }^{(10)}$.

É importante ressaltar que ambas as simulações são realizadas fazendo uso de uma análise dinâmica. Vários autores como por exemplo Nakamachi ${ }^{(22)}$, Prior $^{(12)}$ e Soltani ${ }^{(23)}$, entre outros, realizaram comparações entre os métodos implícito e explícito de integração no tempo. Estes autores fizeram comparações entre o método implícito estático com o método explícito dinâmico, desprezando assim a matriz de massa na análise implícita. Observa-se que o método explícito é menos custoso e mais amigável de ser utilizado que o método implícito dinâmico para estes casos (modelos).

\subsection{Analise de Sensibilidade}


Em virtude dos menores tempos de processamento consumidos pelo método explícito de integração no tempo, é avaliada a sensibilidade dos modelos a certos fatores dinâmicos. Visando, assim, uma possível otimização dos modelos.

Deformação crítica ou ponto de maior afinamento é o local onde a chapa conformada apresenta maior afinamento e por conseqüência uma maior possibilidade de apresentar falha neste ponto. No ensaio de estiramento a chapa tende a romper-se no vão livre próximo ao raio do punção, pois este local é o ponto de força de atrito máximo ${ }^{(24)}$. Contudo, no processo de estampagem o ponto onde ocorre a deformação crítica é localizado na região da parede do copo bem próximo ao raio de adoçamento do punção ${ }^{(25)}$.

\subsubsection{Sensibilidade à Velocidade}

\section{a) Sensibilidade à Velocidade numa Análise de Estiramento}

Para se avaliar a sensibilidade do modelo ao aumento da velocidade, imposta ao punção, são realizadas vinte e duas simulações com velocidades que variam de $0,10 \mathrm{~m} / \mathrm{s}$ à $40 \mathrm{~m} / \mathrm{s}$. O modelo apresenta uma elevada sensibilidade ao aumento da velocidade impostas ao mesmo (punção), entretanto as curvas apresentam sempre o mesmo formato. Uma melhor avaliação da sensibilidade do modelo ao aumento da velocidade imposta ao punção é apresentado na Figura 3a, onde apenas os pontos de maior afinamento com suas respectivas localizações (coordenadas) são apresentados. Pode-se observar que a medida que a velocidade do punção aumenta ocorre um aumento considerável na espessura do ponto de maior afinamento. Este fato já foi observado por Wang et $a l^{(26)}$ e por Hsu et al ${ }^{(27)}$. Eles observaram através de um ensaio bastante semelhante ao ensaio de estiramento que a medida que a velocidade de carga do punção aumenta ocorre uma redução no nível de deformações atingidas. Observa-se também que a maior influência da velocidade, neste caso, ocorre para valores entre 0,1 e $10 \mathrm{~m} / \mathrm{s}$, pois nesta faixa de velocidade a deformação crítica apresenta um aumento de $10,22 \%$. Já para valores de velocidade que variam entre 10 e $20 \mathrm{~m} / \mathrm{s}$ o aumento é de apenas 2,21\% no valor da espessura no ponto de maior afinamento.

Outro fato bastante importante é que para valores de velocidade máxima superiores a $20 \mathrm{~m} / \mathrm{s}$ o modelo passa a não ser mais confiável. Pois para velocidades superiores a $20 \mathrm{~m} / \mathrm{s}$, neste caso alguns elementos se degeneram tornando assim a resposta não confiável. Para estes valores de velocidade, superiores a $20 \mathrm{~m} / \mathrm{s}$, a energia cinética média ficou superior a $5 \%$ da energia de deformação média. Fazendo uma análise no tempo, a energia cinética é superior a 5\% da energia de deformação mais de 55\% do tempo total da análise.

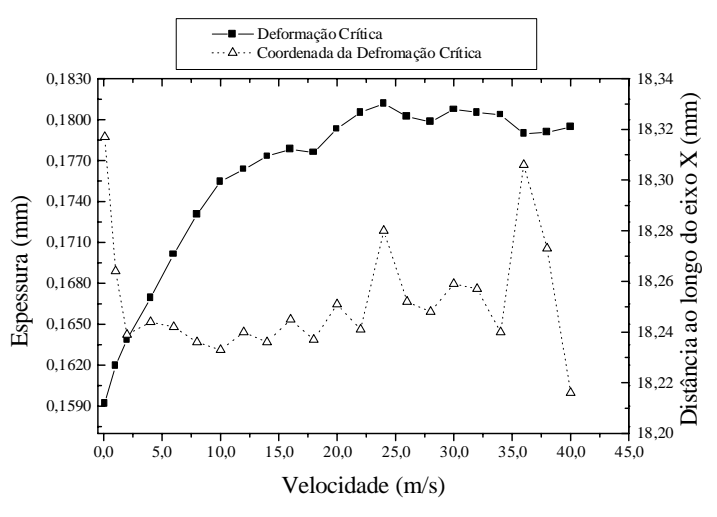

(a)

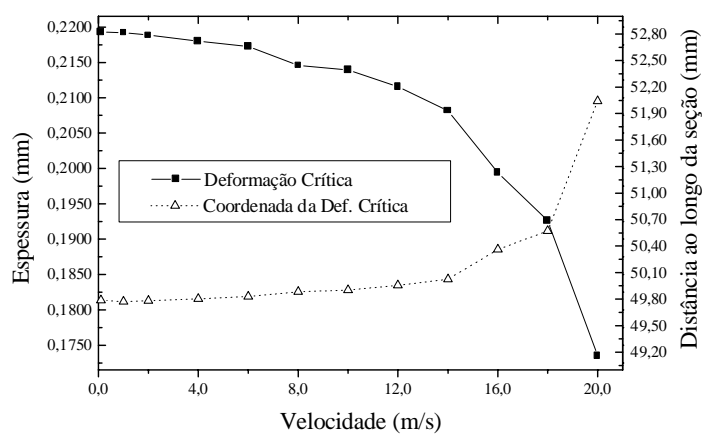

(b) 
Figura 3: Variação do ponto de maior afinamento com o aumento da velocidade:

(a) Ensaio de estiramento;

(b) Processo de estampagem.

\section{b) Sensibilidade à Velocidade numa Análise de Estampagem}

Assim como no modelo anterior, são realizadas vinte e duas simulações com velocidades que variam de $0,10 \mathrm{~m} / \mathrm{s}$ à $40 \mathrm{~m} / \mathrm{s}$. Observa-se que o modelo apresenta uma elevada sensibilidade ao aumento da velocidade impostas ao modelo (ferramental), mas as curvas apresentam sempre o mesmo formato. Na Figura $3 b$ pode-se avaliar melhor a sensibilidade do modelo ao aumento da velocidade imposta ao punção, na região de deformação crítica.

Observa-se que a medida que a velocidade do punção aumenta ocorre uma considerável redução na espessura do ponto de deformação crítica (ponto de maior afinamento). A maior influência da velocidade, neste caso, ocorre para valores entre 10 e $20 \mathrm{~m} / \mathrm{s}$ pois nesta faixa de velocidade a deformação crítica apresenta uma redução em seu valor de $19,0 \%$. Para valores de velocidade que variam entre 0,1 e $10 \mathrm{~m} / \mathrm{s}$ a redução é de apenas $2,4 \%$ no valor da espessura do ponto de maior afinamento. Pode-se observar também que a maior influência da velocidade na localização do ponto de deformação crítica, neste caso, é para valores entre 14 e $20 \mathrm{~m} / \mathrm{s}$, onde ocorre um aumento na coordenada do ponto de deformação crítica de 4,0\%. Apesar de serem realizadas simulações com a velocidades variando de 22 a $40 \mathrm{~m} / \mathrm{s}$, seus respectivos resultados não são apresentados, pois para velocidades acima de $20 \mathrm{~m} / \mathrm{s}$ alguns elementos que compõem a malha da chapa se degeneram tornando os resultados das análises não confiáveis.

\section{c) Disposições Finais}

Conforme pode-se observar tanto para o modelo de estiramento quanto para o modelo de estampagem, para velocidades superiores a $20 \mathrm{~m} / \mathrm{s}$ os resultados passam a não ser mais confiáveis. Mas para níveis de velocidade inferiores a $20 \mathrm{~m} / \mathrm{s}$, em nenhum dos modelos, a energia cinética média ultrapassou $4 \%$ da energia de deformação média. Vários autores, tais como Prior $^{(12)}$ e Rebelo et al $^{(28)}$, têm chamado a atenção para os efeitos causados por elevados níveis de velocidade atingidas pela análise e por conseqüência elevados níveis de energia cinética. Eles têm tentado estabelecer critérios práticos para se evitar o efeito da velocidade nos resultados fornecidos pelo modelo. Rebelo et $a l^{(28)}$ sugeriram que velocidades entre 15 e $20 \mathrm{~m} / \mathrm{s}$ poderiam ser utilizadas numa análise de conformação, entre elas na estampagem, sem que os efeitos inerciais fossem percebidos. Já Prior $^{(12)}$ sugere que a energia cinética não deve ultrapassar $5 \%$ da energia de deformação do modelo para evitar o comprometimento dos resultados simulados.

Mas conforme observado pelas análises realizadas, tanto no que se refere ao estiramento quanto à estampagem, é praticamente impossível evitar que no início da análise o valor da energia cinética seja inferior a $5 \%$ da energia de deformação. Este fato pode ser observado facilmente na Figura 4, onde é apresentada a evolução da relação $E c / E d e f$ ao longo do tempo de análise, para níveis de velocidades considerados muito baixos. Segundo Prior ${ }^{(12)}$, análises em que a velocidade atingida pelo ferramental é inferior da $10 \mathrm{~m} / \mathrm{s}$ podem ser consideradas como sendo análises quase-estáticas. E em alguns casos a energia cinética ficou superior a $5 \%$ da energia de deformação mais de $50 \%$ do tempo total da análise, antes que o modelo se degenerasse. Em virtude de tais fatos apresentados, pode-se concluir que um estudo mais aprofundado dos efeitos da velocidade imposta ao modelo, efeitos de inércia, 
devem ser realizados. Pois os critérios e parâmetros de limite de velocidade e energia cinética sugeridos pela literatura. ${ }^{(12,13,28)}$ mostraram ser, para estes modelos simulados, bastante ineficazes conforme observado.

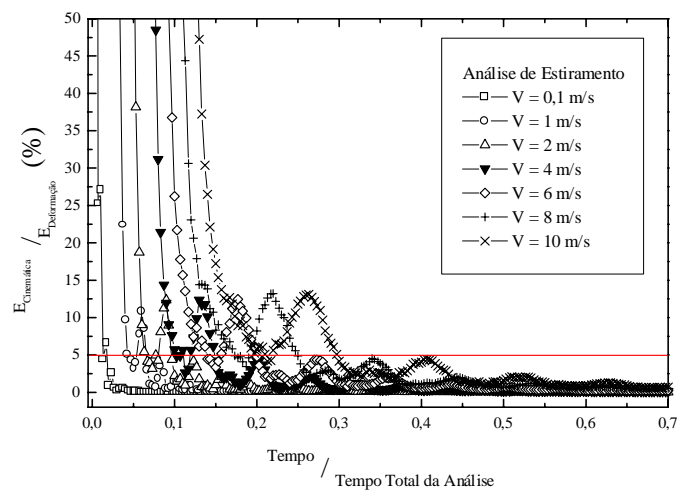

(a)

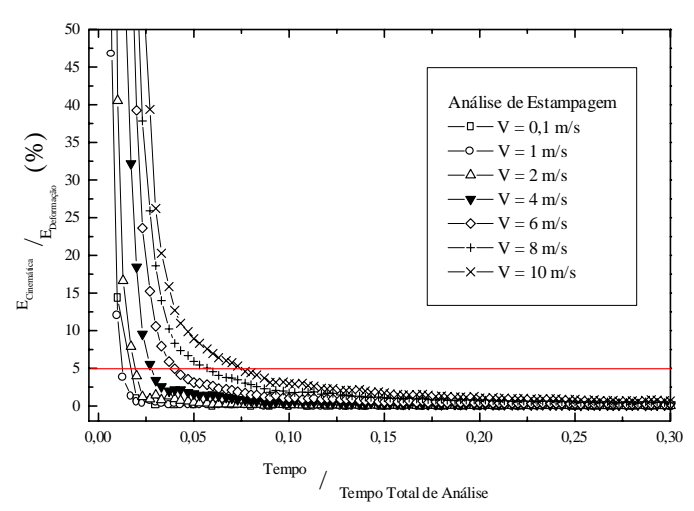

(b)

Figura 4: Variação da Ec/Edef com o aumento da velocidade:

(a) Ensaio de estiramento;

(b) Processo de estampagem.

\subsubsection{Sensibilidade ao Refinamento da Malha}

Para se avaliar a sensibilidade do modelo de estampagem são apresentadas doze simula

ções com características de refinamento diferentes, ou seja com diferentes tamanhos de elementos. É observado que qualitativamente, assim como nos casos anteriores, as curvas de espessura quando comparadas com os valores experimentais medidos apresentavam o mesmo formato. Em virtude deste fato, são apresentados a seguir apenas os valores dos pontos de maior afinamento (deformação crítica) conforme apresentado na Figura 5.

Inicialmente, pode-se observar que para o modelo de contato existente no ABAQUS/EXPLICIT, onde o ferramental é composto de elementos rígidos que formam uma superfície rígida (master) que entra em contato com a chapa (slave), o refino da malha do ferramental praticamente não apresenta influência na acurácia dos resultados obtidos pela simulação. O fato do refino da malha no ferramental não ter influência pode ser explicado pela forma na qual é estabelecida e caracterizada o contato no ABAQUS/EXPLICIT. Neste caso, o código utiliza uma formulação master-slave "estimada". Nesta formulação as restrições de contato são aplicadas de modo que não ocorra penetração em ambas as superfícies, ou seja o contato é forçado de modo que as duas superfícies façam o papel de superfície master e slave ao mesmo tempo. Impedindo, assim, que os nós da superfície slave, como em geral ocorre, penetre nos segmentos da superfície master. As restrições de contato são desta forma uma média das duas restrições master-slave ${ }^{(18,19)}$. 


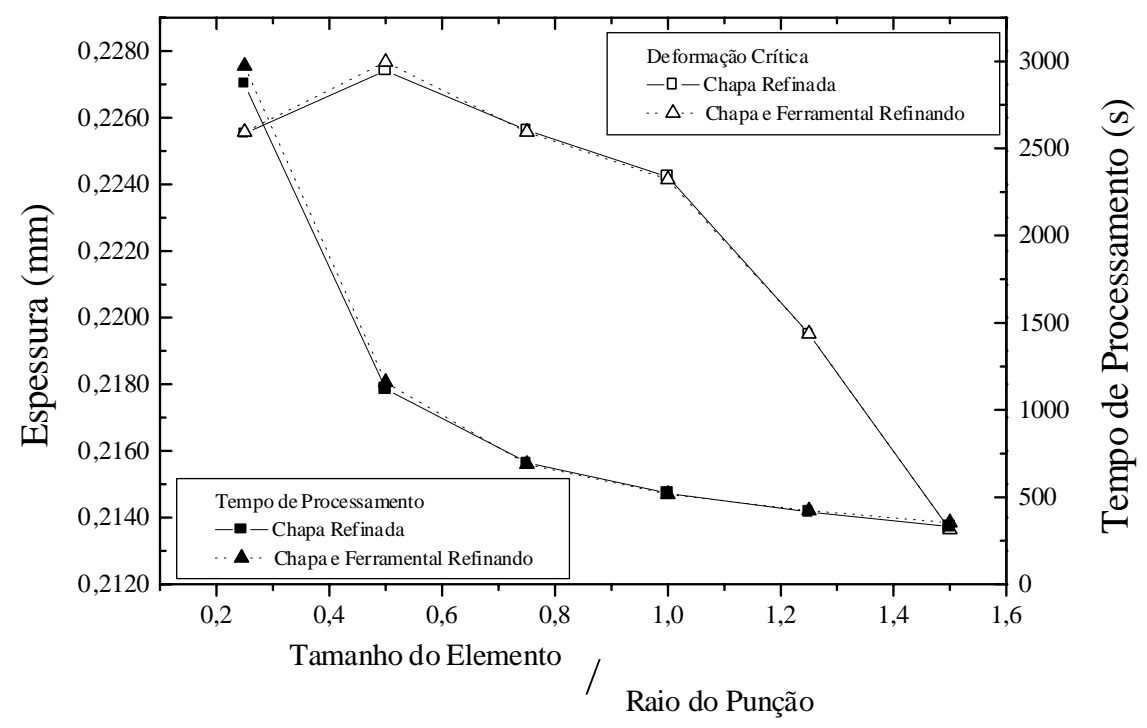

Figura 5: Variação do ponto de maior afinamento e do tempo de processamento com o refino da malha no processo de estampagem.

Deve-se notar também que o efeito do refinamento da malha sobre a deformação crítica apresenta um formato parabólico decrescente, cujo melhor valor ocorre para $\mathrm{Tel} / \mathrm{Rp}=0,5$, onde o valor da deformação crítica é de 0,2241 $\mathrm{mm}$. Este valor ideal de relação entre o tamanho elemento e o raio de adoçamento do punção (menor raio existente na geometria do modelo) também foi obtido por Mattiason et al $^{(17)}$. Por outro lado, o efeito do refinamento da malha sobre o tempo de processamento (CPU Time) apresenta um formato exponencial decrescente. Para o valor ideal da relação $\mathrm{Tel} / \mathrm{Rp}=0,5 \mathrm{um}$ aumento de 3 vezes no número de elementos da chapa leva a um aumento de $6,5 \%$ no valor da deformação crítica, conforme apresentado na Figura 5. Este aumento de 3 vezes no número de elementos da chapa também leva a um aumento de 3,4 vezes no tempo de processamento e de 3 vezes no número de incrementos.

\section{Conclusões}

Após terem sido realizadas aproximadamente cem simulações, consumindo um total de cem horas de processamento em um supercomputador CRAY J90. Os resultados obtidos nas análises de sensibilidade dos modelos de ensaio de estiramento e do processo de estampagem da lata de atum através de simulações numéricas pelo uso do método de elementos finitos (MEF), empregando-se o código comercial ABAQUS, e pela comparação com os resultados experimentais obtidos por Moreira $^{(2)}$ e Villas Boas ${ }^{(21)}$ permitiram as seguintes conclusões:

1. Para ambos os modelos simulados o método implícito dinâmico mostrou ser mais estável, porém computacionalmente mais custoso. Sendo que, no caso do modelo de estampagem os métodos implícito e explícito praticamente obtiveram coincidência de resultados simulados. 
2. O método explícito dinâmico mostra ser mais amigável e menos custoso de ser utilizado que o método implícito dinâmico, apresentando assim uma melhor relação custo/benefício, nos modelos simulados.

3. Tanto o modelo de ensaio de estiramento quanto o modelo de estampagem da lata de atum, nos casos simulados, mostram ser bastante sensíveis à velocidade imposta ao ferramental, quando comparados os resultados simulados com os dados experimentais.

4. Para velocidades superiores a $20 \mathrm{~m} / \mathrm{s}$, os resultados obtidos pelas simulações, para ambos os modelos, passam a não ser confiáveis. Pois, para os casos simulados com velocidades superiores a $20 \mathrm{~m} / \mathrm{s}$, alguns elementos dos modelos se degeneram.

5. Em ambos os modelos simulados, mesmo para velocidades muito baixas $(0,1 \mathrm{~m} / \mathrm{s})$ quando análise pode ser considerada quase-estática, a energia cinética é superior a $5 \%$ da energia de deformação durante parte do tempo de evolução da análise.

6. O modelo de estampagem simulado, mostra ser bastante sensível ao refinamento da malha da chapa, mas mostra ser insensível ao refinamento da malha do ferramental, neste caso. Onde o modelo obtêm uma maior acurácia de resultados para a relação tamanho de elemento da chapa sobre o tamanho do menor raio do ferramental igual a 0,5.

\section{Referências}

[1] Dieter, George - Metalurgia Mecânica, $2^{\mathrm{a}}$ Edição, Editora Guanabara Koogan S. A., Rio de Janeiro, 1981, 654 p. Seção 20.5: Estiramento, pp 583 - 586.

[2] Moreira, Luciano - "Desenvolvimento e Simulação por Elementos Finitos de um Ensaio de Estiramento de Chapas Finas”, Tese de Mestrado - EEIMVR/UFF, Volta Redonda, Agosto de 1996.

[3] Miles, M. P.; Siles, J. L.; Wagner, R. H.; Narasimhan, K. - "A Better Sheet Formability Test", A Metalurgical Transactions, Vol. 24A, pp.1143-1151, 1993.

[4] Wagoner, R. H.; Wang, W.; Sriram, S. - "Development of OSU Formability Teste and OSU Friction Test”, Journal of Materials Processing Technology - Elsevier Science B. V., Vol. 45, pp 13 - 18, 1991.

[5] Hosford, Wilian F.; Caddel, Robert M. - Metal Forming: mechanics and Metallurgy, $2^{\mathrm{a}}$ Edition, Edited by Prentice Hall Inc., Englewood Cliffs, New Jersy, 1983, 364p. Cap. 14: Cupping, Redrawing, and Ironing, pp 286 - 308.

[6] Dieter, George - Metalurgia Mecânica, 2a Edição, Editora Guanabara Koogan S. A., Rio de Janeiro, 1981, 654 p. Seção 20.6: Estampagem Profunda ou Embutimetno, pp 587 593.

[7] Backofen, Walter A. - Deformation Processing. London: Addison - Wesley Publishing Company, 1972. 326p. Cap. 11: Drawability, pp227 - 241.

[8] Menezes, M. A. - "Strain Limit Theories, Anisotropy in Sheet Metal Forming and Simulation of Pressing Processes”, Tese de Doutorado - University of Birminghan, Agust 1995. 
[9] Karl Schweizerhof, K.; Hallquist, J. O. - "Explicit Time Integration and Contact Simulations for Thin Sheet Metalforming”, International Conference FE-Simulation of 3D Sheet Metal Forming Processes in Automotive Industry, Zurich, May 1991.

[10] ABAQUS - Theory Manual, Version 5.4 - Hibbitt, Karlsson \& Sorenson Inc., item 2.4 .

[11] Rebelo, N.; Nagtegaal, J. C.; Taylor, L. M. - "Comparasion of Implicit and Explicit Finite Element Methods in the Simulation of Metal Forming Processes”, Numerical Methods in Industrial Forming Process, pp 99 - 108, Rotterdam, 1992.

[12] Prior, A. M. - "Applications of Implicit and Explicit Finite Element Techniques to Metal Froming”, Journal of Materials Processing Technology - Elsevier Science B.V., pp 649 656, 1994.

[13] Haug, E.; Di Pasquale, E.; Pickett, A. K.; Ulrich, D. - "Industrial Sheet Metal Forming Simulation Using Explicit Finite Element Methods", International Confernce FESimulation of 3D Sheet Metal Forming in Automotive Industry, Zurich, May 1991.

[14] Cook, R. D. - Concepts and Applicatiuons of Finite Element Analysis, Edited by John Willey e Sons, Third Edition, pp 376 - 429, New York 1989.

[15] ANSYS User’s Manual Revision 5.0 - “Theory Manual - Vol. IV”, ANSYS Inc., pp. 171 - 17-48, December 1992.

[16] Burford, D. A., Narasimhan, K.; Wagoner, R. H. - “A Theoretical Sensitivity Analysis for Full-Dome Formability Tests: Parameter Study for n, m ,r, and ”, A Metallurgical Transactions, Vol. 22A, pp 1775-1788, august - 1991.

[17] Mattiason, K. - BEMSPANG, L. \& SAMUELSSON - HAMMAN, T.; SCHEDIN, E. \& MELANDER, A. - "Evaluation of a Dynamic approach Using Explicit Integration in 3-D Sheet Forming Simulation”, Numerical Methods in Industrial Forming Process, pp 55-66, Rotterdam, 1992.

[18] ABAQUS User's Manual - Volume I, Version 5.4 - Hibbitt, Karlsson \& Sorenson Inc., item 5.2.1, pp 5.2.1-1 - 5.2.1-5.

[19] ABAQUS User's Manual - Volume II, Version 5.4 - Hibbitt, Karlsson \& Sorenson Inc., item 8.4.15, pp 8.4.15-1 - 8.4.15-2.

[20] ABAQUS User's Manual - Volume I, Version 5.4 - Hibbitt, Karlsson \& Sorenson Inc., item 8.7.3, pp 8.7.3-1 - 8.7.3-10.

[21] Villas Boas, Eduardo - "Influência da Utilização de Materiais com Dupla Redução Sobre à Embutibilidade de Produtos com Geometria Cilíndrica”, Tese de Mestrado EEIMVR/UFF, Volta Redonda, Maio de 1996.

[22] Wagner, R. H.; Nakamachi, E.; Germain, Y. - "Experience with Explicit and Implicit Finite Element Programs for Sheet Forming Analysis", Modeling of Metal Forming Processes, pp 131 - 138, France, August, 1988. 
[23] Soltani, B.; Mattiasson, K.; Samuelsson, A. - "Implicit and Dynamic Explicit Solutions of Balde Forming Using the Finte Element Method”, Journal of Materials Processing Technology - Elsevier Science B.V., vol. 54, pp 69 - 74, 1994.

[24] LANCELLOTTI, MARLOS ROBERTO - "Simulação de Ensaio de Estiramento por Elementos Finitos Utilizando uma Técnica Estática”, Tese de Mestrado - EEIMVR/UFF, Volta Redonda, Março de 1998.

[25] França Júnior, Hélio DOS SANTOS - "Sensibilidade da Simulação Numérica à Variação de Propriedades do Material e Parâmetros do Processo de Conformação de Chapas”, Tese de Mestrado - EEIMVR/UFF, Volta Redonda, Março de 1998.

[26] Wang, W.; Wagoner, Robert H. - "A Realistic Friction Teste for Sheet Forming Operations", Sheet Metal and Stamping Symposium / SAE SP-994, pp 223 - 230, Warrendale, March 1993.

[27] Tze-Chi Hsu; Kuo, S. - "Boundary Friction Model in Punch Friction Test”, Journal of Materials Processing Technology - Elsevier Science B.V., pp 601 - 606, 1994.

[28] Rebelo, N.; Nagtegaal, J. C.; Hibbitt, H. D. - "Pratical Apects of Modeling Sheet Forming Processes”, Numiform 89, pp 31-43, Rotterdam, 1989.

\section{Informações bibliográficas:}

Conforme a NBR 6023:2002 da Associação Brasileira de Normas Técnicas (ABNT), este texto científico publicado em periódico eletrônico deve ser citado da seguinte forma: PALMEIRA, A. A.; HABIBE, A. F.; JÁCOME, P. A. D. . Técnicas de Modelagem de Conformação de Chapas por Elementos Finitos. Cadernos UniFOA , Volta Redonda, ano 1, $\mathrm{n}^{\circ}$. 1, jul. 2006. Disponível em: $<$ http://www.unifoa.edu.br/pesquisa/caderno/edicao/01/41.pdf > 